\title{
Heterogeneous Adsorption and Local Ordering of Formate on a Magnetite Surface
}

\author{
Marcus Creutzburg, ${ }^{\perp}$ Kai Sellschopp, ${ }^{\perp}$ Steffen Tober, Elin Grånäs, Vedran Vonk, \\ Wernfried Mayr-Schmölzer, Stefan Müller, Heshmat Noei, Gregor B. Vonbun-Feldbauer,* \\ and Andreas Stierle*
}

Cite This: J. Phys. Chem. Lett. 2021, 12, 3847-3852

Read Online

ACCESS | 네 Metrics \& More | 回 Article Recommendations | sl Supporting Information

ABSTRACT: We report a novel heterogeneous adsorption mechanism of formic acid on the magnetite (111) surface. Our experimental results and density functional theory (DFT) calculations give evidence for dissociative adsorption of formic acid in quasibidentate and chelating geometries. The latter is induced by the presence of iron vacancies at the surface, making oxygen atoms accessible for hydrogen atoms from dissociated formic acid. DFT calculations predict that both adsorption geometries are energetically favorable under our experimental conditions. The calculations prove that the locally observed $(\sqrt{3} \times \sqrt{3}) R 30^{\circ}$ superstructure consists of three formate molecules in a triangular arrangement, adsorbed predominantly in a chelating geometry. The results show how defects can stabilize alternative adsorption geometries, which is a crucial ingredient for a detailed atomistic understanding of reaction barriers on magnetite and other oxide surfaces, as well as for the stability of carboxylic acid based nanocomposite materials.

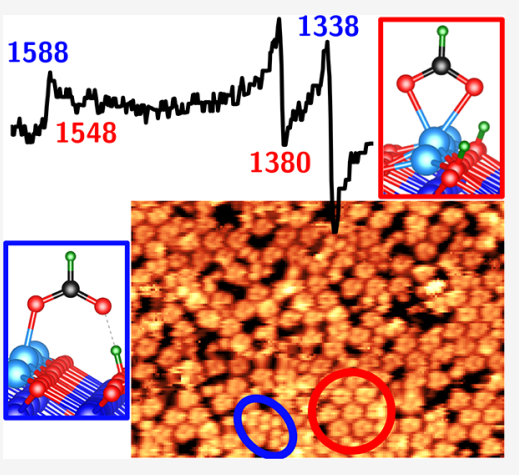

$\mathrm{M}$ agnetite $\left(\mathrm{Fe}_{3} \mathrm{O}_{4}\right)$ is a widely used transition metal oxide with applications in catalysis ${ }^{1}$ such as the FischerTropsch synthesis ${ }^{2}$ and the water-gas shift reaction. ${ }^{3}$ Formic acid $(\mathrm{HCOOH})$ as the elementary carboxylic acid is proposed to occur as an intermediate during these reactions; therefore, an atomistic understanding of its interaction with magnetite surfaces is vital. $^{3-5}$ Organic acids, exhibiting the same carboxylic end group as formic acid, are widely employed as "binders" in a new class of bottom up fabricated nanocomposite materials. ${ }^{6}$ Since reducible oxides, such as magnetite, show a complex defect chemistry, the role of surface defects for the adsorption behavior of organic molecules is of special importance. ${ }^{7,8}$ For magnetite, the (001) and the (111) surfaces are of high significance, because of their thermodynamical stability and consequently their predominant occurrence as nanoparticle facets. ${ }^{9}$ Considerable progress has been made recently in the understanding of the formic acid adsorption on magnetite (001). Formic acid is reported to dissociatively adsorb at room temperature ( $\mathrm{rt}$ ) in a bidentate bridging geometry predominantly in a specific adsorption site with both oxygens bound to two octahedral surface iron ions. ${ }^{10,11}$ In contrast, on other metal oxide surfaces heterogeneous adsorption of formic acid at rt was observed. Bidentate bridging and monodentate geometries were reported on anatase $\mathrm{TiO}_{2}(101) .^{12}$ On $\mathrm{ZnO}(10 \overline{1} 0)$, bidentate bridging and quasibidentate (i.e., one formate oxygen bound to a metal atom, the other one to an $\mathrm{OH}$ group on the surface) configurations were identified, oriented along different surface directions. ${ }^{13}$ Studies of the interaction of formic acid with the magnetite (111) surface, on the other hand, are scarce. An STM study of formate on magnetite (111) suggested bidentate chelating adsorption at low coverage on iron-terminated surface areas based on the asymmetric appearance of the molecules in the images. ${ }^{14}$ Other oxygen-terminated areas of the crystal were found to be inert to adsorption.

The atomic structure of the clean magnetite (111) surface exhibits characteristic defects depending on the preparation conditions; ${ }^{15}$ it is, however, unclear how the defects influence molecular adsorption behavior. A perfect or defective $\mathrm{Fe}_{\text {tet1 }}$ termination with a hexagonal array of tetrahedral iron ions was observed experimentally on magnetite thin films grown on $\mathrm{Pt}(111)$, as well as on magnetite single crystals. ${ }^{16-18}$ On single crystals, the $\mathrm{Fe}_{\text {tet1 }}$ termination was also reported to coexist with the $\mathrm{Fe}_{\text {oct2 }}$ termination, ${ }^{18,19}$ with the latter predicted to be more stable under highly reducing conditions $\mathrm{s}^{20}$ and after adsorption of $\mathrm{CO}^{21}$ and water. ${ }^{2-24}$ In this study, we apply complementary Fourier transform infrared reflection absorption spectroscopy (FT-IRRAS), STM, low energy electron diffraction (LEED), and surface X-ray diffraction (SXRD) to investigate the adsorption of formic acid on the clean, welldefined $\mathrm{Fe}_{\text {tet1 }}$-terminated magnetite (111) single crystal surface

Received: January 20, 2021

Accepted: March 24, 2021

Published: April 14, 2021 
with controlled $\mathrm{Fe}_{\text {tet1 }}$ vacancies. We obtain detailed information on the adsorption geometries influenced by the surface cation vacancies, as well as intermolecular interactions. Density functional theory (DFT) calculations and calculated IR spectra allow a rigorous interpretation of our experimental data and a prediction of the most stable adsorption geometries.

The clean magnetite (111) single crystal surface was prepared by multiple cycles of Ar ion sputtering and annealing (see Experimental Section in the Supporting Information (SI)). Under these conditions, we find that the surface is $~ 80 \%$ $\mathrm{Fe}_{\text {tet1 }}$-terminated, which we conclude from the quantitative analysis of the SXRD data (see Figures S3 and S4). Other terminations, e.g., $\mathrm{Fe}_{\text {oct2 }}$, result in higher SXRD goodness-of-fit values and can therefore be ruled out, see Table S2. Figure 1a shows a side view of the atomic layers stacked along the [111] direction. The relative occupancies of the layers from the surface to the bulk as determined by SXRD are displayed in Figure 1a; relaxations given in Figure S4 agree well with LEED

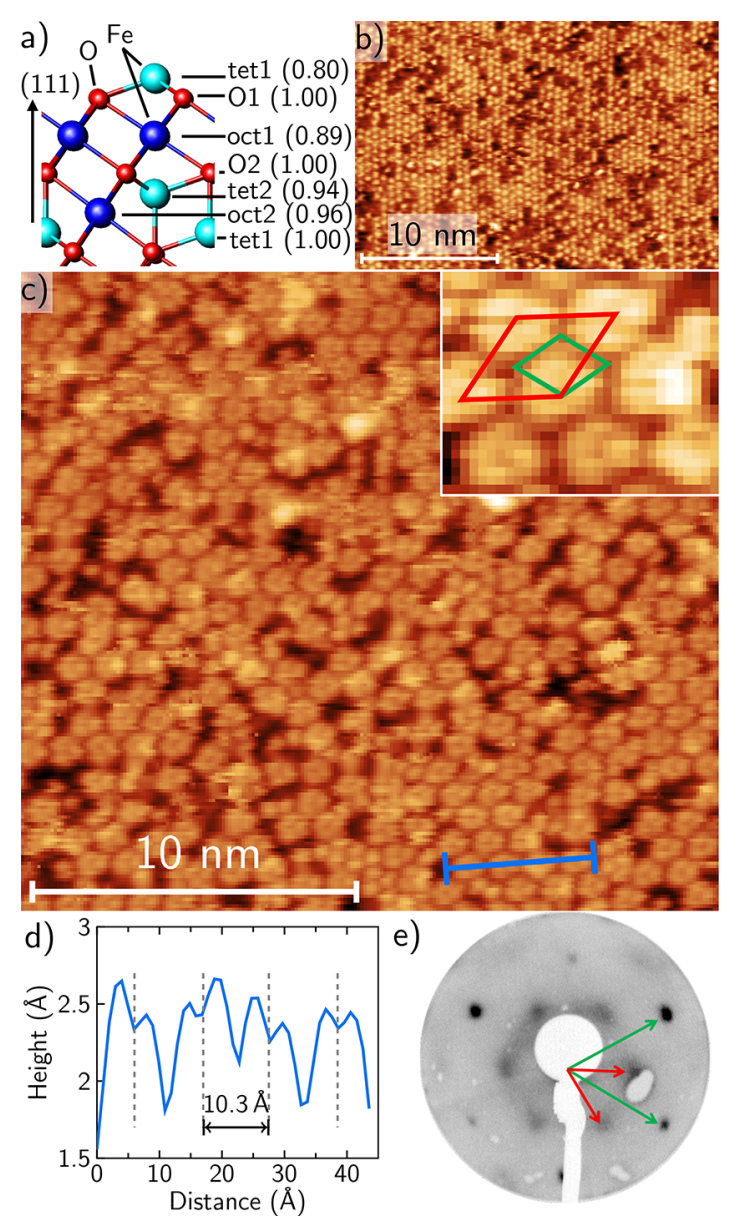

Figure 1. (a) Side view of magnetite (111) with labeled terminations. Atomic occupancies obtained from the SXRD fit are given in parentheses (error bar $\pm 5 \%{ }^{26}$ ). (b) STM image of the clean magnetite (111) surface at rt. Tunneling parameters: $-1.5 \mathrm{~V}, 1.1$ nA. (c) STM image of magnetite (111) after exposure to $10 \mathrm{~L}$ formic acid at rt. Tunneling parameters: $0.5 \mathrm{nA}, 1 \mathrm{~V}$. Inset: $3 \times 2.5 \mathrm{~nm}^{2} \mathrm{STM}$ image with indicated unit cells of the superstructure (red) and substrate (green). (d) Line scan through the hexagonal superstructure (blue line in panel c). Dashed lines indicate the center of the round features. (e) LEED pattern taken at $19 \mathrm{eV}$ after an exposure to $10 \mathrm{~L}$ formic acid. Reciprocal unit cell vectors are indicated for the $(\sqrt{ } 3 \times$ $\sqrt{ } 3) R 30^{\circ}$ superstructure (red) and the substrate (green).
I(V) results. ${ }^{16,17}$ The STM image presented in Figure $1 \mathrm{~b}$ exhibits the characteristic hexagonal $5.9 \AA$ periodicity of the $\mathrm{Fe}_{\text {tet1 }}$ termination with $\mathrm{Fe}_{\text {tet }}$ as bright spots (see Figure S1). Tetrahedral $\mathrm{Fe}$ vacancies are visible in line with the SXRD results, and the locally oxygen O1-terminated surface appears dark in the STM image at negative bias voltage. ${ }^{25}$ Iron vacancies are characteristic defects in the $\mathrm{Fe}_{3} \mathrm{O}_{4}(111)$ top surface layer. $^{16,20,25}$ The step height of $4.9 \pm 0.2 \AA$ measured by STM represents the repeat distance of the $\mathrm{Fe}_{\text {tet } 1}$ termination along the $\mathrm{Fe}_{3} \mathrm{O}_{4}[111]$ direction. It proves that the termination does not change across terraces, and that it is uniform over the whole crystal after this preparation, see Figure S2.

The clean surface was exposed to 10 Langmuir $(1 \mathrm{~L}=1.33$ $\times 10^{-6}$ mbar s $)$ formic acid $\left(2 \times 10^{-7}\right.$ mbar, $\left.66 \mathrm{~s}\right)$ at $\mathrm{rt}$. The LEED pattern recorded after adsorption (Figure 1e and Figure S5) shows the first order diffraction spots of magnetite (111) and additional diffraction spots evidencing the formation of a weakly ordered $(\sqrt{3} \times \sqrt{3}) R 30^{\circ}$ superstructure. The STM image (Figure 1c) was recorded after exposure to $10 \mathrm{~L}$ formic acid. The surface is partly covered by large bright features arranged in a hexagonal superstructure. Their closer inspection reveals that they are made up by three smaller features in a triangular arrangement. An STM line scan (Figure 1d) shows a superstructure periodicity of $10.3 \pm 0.5 \AA$. The internal structure of the features gives rise to a distinct splitting of the line scan maxima. We interpret the bright features observed at positive bias voltage as ontop hydrogen atoms of formic acid. ${ }^{11}$ Thus, we conclude that the hexagonally arranged features in the $(\sqrt{3} \times \sqrt{3}) R \quad 30^{\circ}$ superstructure consist of three dissociated formic acid molecules (as we will show below) clustered together in a structural motif with 3-fold symmetry with hydrogen atoms being $\sim 4 \AA$ apart, see the inset to Figure 1c. In addition to the superstructure, there are bare areas (dark) and several smaller bright protrusions that we assign to formic acid in a different adsorption site. The lack of the superstructure's long-range order is also reflected in the LEED patterns with rather broad diffraction spots. The step height of $4.9 \AA$ remains unchanged after formic acid adsorption compared to the clean surface, see Figure S6.

FT-IRRAS measurements were performed to elucidate the binding geometry of formic acid on magnetite (111), see SI for details. Formic acid, $2 \mathrm{~L}\left(2 \times 10^{-7} \mathrm{mbar}, 13 \mathrm{~s}\right)$, was dosed to the clean surface at rt. We observe prominent vibrational bands at 1338,1380 , and $1588 \mathrm{~cm}^{-1}$ and a weak band at $1548 \mathrm{~cm}^{-1}$ (see Figure 2, full spectrum available in Figure S7). Molecular

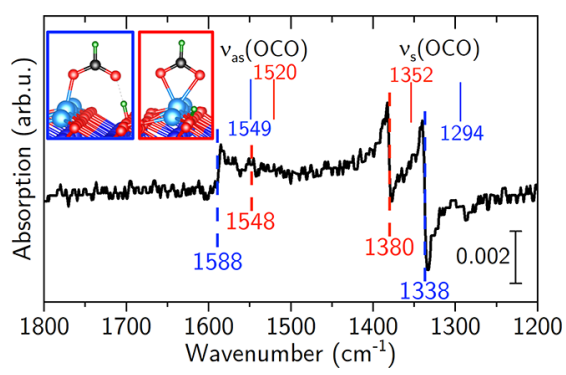

Figure 2. p-polarized FT-IRRA spectrum for a formic acid coverage of $2 \mathrm{~L}$. Vibrational bands assigned to the quasibidentate geometry are labeled in blue and to the chelating geometry in red (dashed lines indicate band center positions). Calculated IR band positions are shown as blue (for quasibidentate) and red (for chelating) solid vertical lines. The inset shows the adsorption geometries. 
adsorption and monodentate formate can be excluded due to the absence of the $\nu(\mathrm{C}=\mathrm{O})$ vibrational band in the region 1800-1600 $\mathrm{cm}^{-1}$. ${ }^{11}$ The observed vibrational bands are therefore in line with dissociated formic acid. Since the distance between neighboring $\mathrm{Fe}_{\text {tet }}$ ions on this surface is 5.9 $\AA$, a bidentate bridging geometry is excluded. It typically requires a smaller distance of the two cations (e.g., $\sim 2.8 \AA$ for magnetite $\left.(001)^{10}\right)$. On the (001) surface, formic acid adsorbs in a bidentate bridging geometry giving rise to only two vibrational bands at 1544 and $1368 \mathrm{~cm}^{-1}$, which are assigned to the asymmetric and symmetric stretching vibrations, $\nu_{\text {as }}$ and $\nu_{s}(\mathrm{OCO})$, respectively. ${ }^{10,11}$ On the (111) surface, the bands at 1338 and $1588 \mathrm{~cm}^{-1}$ appear, however, at similar positions as reported for the quasibidentate adsorption of formate on $\mathrm{ZnO}(10 \overline{1} 0)$, making this a more likely candidate $\left(\nu_{s}(\mathrm{OCO})\right.$ at $1373 \mathrm{~cm}^{-1}$ and $\nu_{\text {as }}($ OCO $)$ at $\left.1589 \mathrm{~cm}^{-1}\right){ }^{13}$ The smaller splitting of the $\nu_{\mathrm{s}, \mathrm{as}}(\mathrm{OCO})$ bands at 1380 and $1548 \mathrm{~cm}^{-1}$ suggests a smaller $\mathrm{O}-\mathrm{C}-\mathrm{O}$ bond angle, such as a chelating adsorption geometry with both formate oxygen atoms bound to one single $\mathrm{Fe}_{\text {tet } 1}$ ion. ${ }^{27}$ All vibrational bands present a Fano line shape which results from magnetite being neither a perfect metal nor a perfect insulator. ${ }^{10,11,28}$ While both bands at 1338 and $1380 \mathrm{~cm}^{-1}$ present a similar line shape originating from a dynamic dipole perpendicular to the surface, the line shape around $1588 \mathrm{~cm}^{-1}$ is inverted, because it is excited by the electric field component parallel to the surface. ${ }^{10,28}$ This component is smaller and has a lower reflectivity than the outof-plane component which typically results in $\nu_{\text {as }}$ vibrational bands being less intense. ${ }^{28}$ The line shape thus supports our assignment of the vibrational symmetry.

To compare the energetics of different adsorption modes and assign the vibrational bands to them, DFT and density functional perturbation theory (DFPT) calculations were performed using a $(\sqrt{3} \times \sqrt{3}) R 30^{\circ}$ supercell with three adsorbed formic acid molecules (full coverage) in molecular, quasibidentate, and chelating adsorption configurations (see SI for computational details and density of states). The molecular starting geometries converged to very high, i.e., unfavorable energies or even converted to quasibidentate configurations during structural relaxation. Therefore, this adsorption mode is considered to be unstable, in agreement with the experimental results, and was excluded from further analysis. The dissociative adsorption in quasibidentate geometry (see Figure $3 \mathrm{a}$ ) is energetically favorable (adsorption energy of $-1.75 \mathrm{eV}$

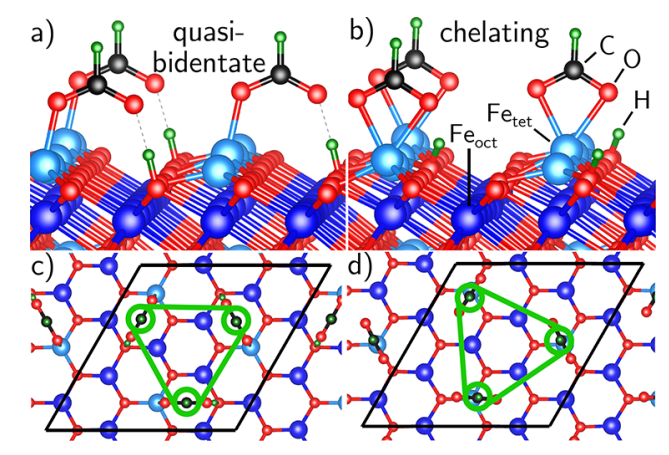

Figure 3. Top and side views of the calculated adsorption structures for quasibidentate $(a, c)$ and chelating $(b, d)$ adsorption of formic acid on magnetite (111). Green circles indicate formate hydrogen imaged by STM. Side views are shown at $1 / 3$ coverage for better visibility. The black rhombus indicates the $(\sqrt{3} \times \sqrt{3}) R 30^{\circ}$ supercell. per molecule at full coverage), with one of the formate oxygens bound to an $\mathrm{Fe}_{\text {tetl }}$ ion. The second is bound via a hydrogen atom originating from dissociation of the molecule to a surface oxygen, at an $\mathrm{O}-\mathrm{C}-\mathrm{O}$ bond angle of $128^{\circ}$. The calculation of the vibrational modes yields a splitting of the symmetric and asymmetric $\mathrm{O}-\mathrm{C}-\mathrm{O}$ stretching vibrations of $254 \mathrm{~cm}^{-1}$, in very good agreement with experiment $\left(250 \mathrm{~cm}^{-1}\right)$. The absolute positions also agree well (see Table 1), taking into account the systematic errors due to the approximations used for the calculations. $^{10,29-31}$

Table 1. Overview of Vibrational Bands of Formic Acid on Magnetite $(111)^{a}$

\begin{tabular}{lcccc} 
& \multicolumn{4}{c}{ wavenumber $\left(\mathrm{cm}^{-1}\right)$} \\
\cline { 2 - 5 } vibrational band & $\exp$ & $\Delta_{\text {exp }}$ & calc & $\Delta_{\text {calc }}$ \\
qbt $\nu_{\mathrm{s}}($ OCO $)$ & 1338 & 250 & 1294 & 254 \\
qbt $\nu_{\text {as }}($ OCO $)$ & 1588 & & 1548 & \\
chel $\nu_{\mathrm{s}}($ OCO $)$ & 1380 & 168 & 1352 & 168 \\
chel $\nu_{\text {as }}($ OCO $)$ & 1548 & & 1520 &
\end{tabular}

${ }^{a}$ The IR spectra were calculated for the chelating (chel) and quasibidentate (qbt) structures in Figure 3. The splittings $\Delta$ of $\nu_{\text {as }}(\mathrm{OCO})$ and $\nu_{\mathrm{s}}(\mathrm{OCO})$ are presented for experimental (exp) and calculated (calc) band positions.

For the chelating adsorption geometry, where both formate oxygen atoms are bound to one $\mathrm{Fe}_{\text {tet }}$ ion, special attention has to be paid to the adsorption site of the dissociated hydrogen. The chelating starting configurations where the dissociated hydrogen was close to the formate also relaxed to a quasibidentate geometry, showing that the quasibidentate configuration is stabilized by a hydrogen bond with a bond length of $1.58 \AA$ to formate $\mathrm{O}$. The chelating configuration only formed when the distance toward the hydrogen was too large to form a hydrogen bond. A thorough investigation of the dependence of the adsorption energy on the distance to the nearest hydrogen can be found in Table S3 and Figure S8. The presence of $\mathrm{Fe}_{\text {tet1 }}$ surface vacancies observed in our experiments however provides an additional degree of freedom to the system, since it makes three additional oxygen atoms per surface unit cell from the $\mathrm{O} 1$ termination available for energetically more favorable hydrogen adsorption (hydrogen adsorption energy of $-2.1 \mathrm{eV}$ on the O1-terminated surface, as compared to $-0.9 \mathrm{eV}$ on oxygen of the $\mathrm{Fe}_{\text {tet } 1}$ termination). Taking into account this possibility of much stronger hydrogen adsorption, a total adsorption energy of $-3.51 \mathrm{eV}$ per formic acid molecule is calculated (see SI for more details). This increase in energetically favorable $\mathrm{H}$ adsorption sites is in line with the STM results in Figure 1c, showing predominantly the triangular motif of the chelating adsorption geometry. In the chelating configuration, the $\mathrm{O}-\mathrm{C}-\mathrm{O}$ bond angle is reduced to $120^{\circ}$. This is also reflected in the reduced splitting of the symmetric and asymmetric $\mathrm{O}-\mathrm{C}-\mathrm{O}$ stretching vibrations of $168 \mathrm{~cm}^{-1}$, perfectly agreeing with the experimental splitting $\left(168 \mathrm{~cm}^{-1}\right)$. The absolute positions again agree well between theory and experiment, see Table 1.

Interestingly, a triangular superstructure (see Figure $3 c, d$ ) is slightly more favorable than a regular high symmetry $(1 \times 1)$ structure for both the quasibidentate and the chelating modes with 10 and $40 \mathrm{meV}$ per molecule, respectively. Therefore, at $\mathrm{rt}$ $(\sim 25 \mathrm{meV})$, the $(\sqrt{3} \times \sqrt{3}) R 30^{\circ}$ superstructure is predominantly formed by the chelating adsorption structure. Furthermore, the orientation of the formates' triangular 
arrangement on top of the hydrogen atoms only matches the chelating structure in a comparison of the STM image and calculated structures from DFT (see green circles in Figure $3 c, d)$. For the calculated quasibidentate structure, the "cluster" of formate molecules is rotated by $30^{\circ}$ and, therefore, does not match the experimental STM images. The heterogeneity of the surface, giving rise to the two adsorption modes, prevents the superstructure from establishing a long-range order on magnetite (111). Further evidence for the assignment of the molecules in chelating geometry to the $(\sqrt{ } 3 \times \sqrt{ } 3) R 30^{\circ}$ reconstructed areas comes from the IR spectra. In the chelating triangular structure, the molecules perform coupled, circular asymmetric stretching vibrations, as visualized in Movies S1 and S2, leading to a suppression of the dynamic dipole moment parallel to the surface. The ratios of calculated IR band intensities for the $\nu_{\text {as }}$ to $\nu_{s}(\mathrm{OCO})$ are 0.051 for the chelating triangular structure and 0.104 for the quasibidentate adsorption geometry, in line with the experimental observation, that the intensity of the chelating asymmetric stretching vibration band at $1548 \mathrm{~cm}^{-1}$ is close to the level of noise (see Figure 2).

To compare the stability of the different binding modes and surface terminations with the experimental conditions, surface stability diagrams were computed from the results of the DFT calculations (see Figure 4, Figure S9 and computational details

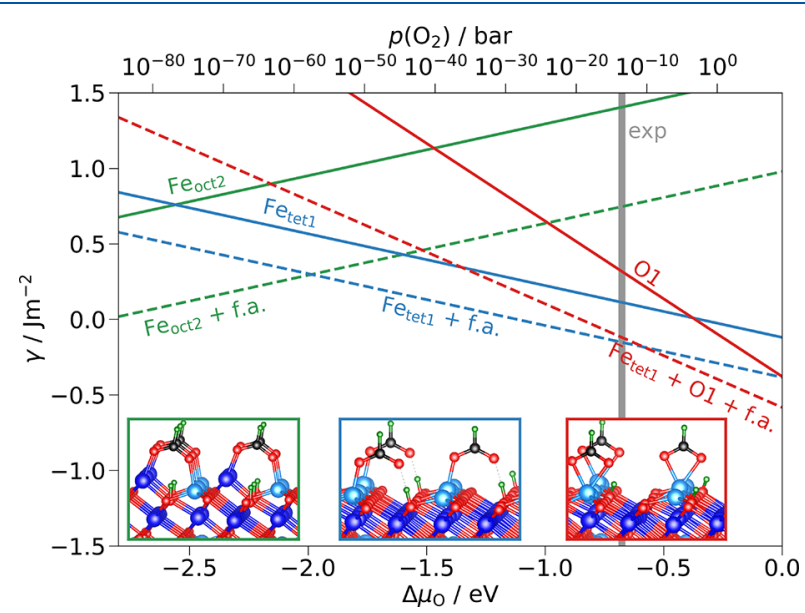

Figure 4. Surface stability diagram showing the surface free energies $\gamma$ (Gibbs free energy per surface area) for different clean surface terminations (solid lines) and adsorption structures (dashed lines) depending on oxygen chemical potential and the corresponding oxygen pressure at $300 \mathrm{~K}^{32}$ The formic acid (f.a.) chemical potential shift with regard to a single molecule in vacuum is fixed to $-1.24 \mathrm{eV}$ equivalent to the experimental pressure of $2 \times 10^{-7}$ mbar at $\mathrm{rt}^{33}$ Green: $\mathrm{Fe}_{\mathrm{oct} 2}$ termination. Blue: $\mathrm{Fe}_{\text {tet1 }}$ termination. Red: $\mathrm{O} 1$ termination and adsorption structure with $50 \% \mathrm{Fe}_{\text {tet1 }}$ and $50 \% \mathrm{O} 1$ terminations (see text). The gray line indicates the experimental conditions. See SI for more information.

in the SI). To assess the whole range of possible conditions, the $\mathrm{Fe}_{\mathrm{oct} 2}, \mathrm{Fe}_{\text {tet } 1}$, and $\mathrm{O} 1$ terminations of magnetite (111) were taken into account. On the $\mathrm{Fe}_{\text {oct2 }}$ termination, formic acid can adsorb in a dissociative bidentate bridging configuration with an adsorption energy of $-2.52 \mathrm{eV}$ per molecule. On the $\mathrm{Fe}_{\text {tet } 1}$ termination, adsorption energies are in the range -1.6 to -2.0 $\mathrm{eV}$ per molecule (see Table S3).

Without formic acid, the $\mathrm{Fe}_{\text {tet1 }}$ termination is predicted to be the most stable under UHV conditions at $\mathrm{rt}$, whereas the oxygen-terminated $\mathrm{O} 1$ structure is more stable at atmospheric oxygen pressure. The difference in adsorption energies leads to an increase of the stability range of the $\mathrm{Fe}_{\text {oct2 }}$ termination at a formic acid pressure of $2 \times 10^{-7} \mathrm{mbar}$ at $\mathrm{rt}$, but the $\mathrm{Fe}_{\text {tet } 1}$ termination is still most stable at UHV typical oxygen pressures. As described before, the heterogeneity of the surface makes it possible for the dissociated hydrogen to bind to oxygen from the O1-terminated parts of the surface (see Figure S10). This combination of terminations with a chelating adsorption of formate at the $\mathrm{Fe}_{\text {tet } 1}$-terminated area is stable at oxygen chemical potentials $>-0.58 \mathrm{eV}$, whereas at less oxidizing conditions a pure $\mathrm{Fe}_{\text {tet } 1}$ termination with a quasibidentate adsorption configuration is favored. Our experimental conditions are close to this chemical potential, explaining why both adsorption structures are present on the surface. The described combination of configurations is likely to form under our experimental conditions, because of the presence of O1-terminated areas on the surface and the mobility of hydrogen on magnetite surfaces at $\mathrm{rt}^{15,34}$

We studied the adsorption behavior of formic acid on the magnetite (111) surface with controlled cation vacancies using complementary methods such as FT-IRRAS, STM, SXRD, LEED, and DFT. Formic acid dissociates on the $\mathrm{Fe}_{\text {tetl }}{ }^{-}$ terminated surface at $\mathrm{rt}$ and adsorbs in two different geometries: quasibidentate and chelating. The latter adsorption configuration is triggered by the presence of iron vacancies at the surface, making oxygen atoms accessible for hydrogen atoms from formic acid. The molecules in the chelating geometry arrange in triangles forming a $(\sqrt{3} \times \sqrt{3}) R 30^{\circ}$ superstructure, pointing out the role of non-negligible intermolecular interactions. DFT calculations of adsorption energies and vibrational frequencies underpin the interpretation of the IR and STM results as originating from the two distinct adsorption geometries. Our results provide evidence that oxide surface defects and heterogeneities may favor otherwise less stable adsorption geometries relevant for the activity of industrial oxide catalysts and the mechanical strength of organic acid based nanocomposite oxide materials.

\section{ASSOCIATED CONTENT}

\section{Supporting Information}

The Supporting Information is available free of charge at https://pubs.acs.org/doi/10.1021/acs.jpclett.1c00209.

Movie showing asymmetric stretching mode $\nu_{\text {as }}(\mathrm{OCO})$ of chelating formate, side view (MP4)

Movie showing asymmetric stretching mode $\nu_{\text {as }}(\mathrm{OCO})$ of chelating formate, top view (MP4)

Experimental and computational methods; clean surface STM images and SXRD data; additional LEED images and full FT-IRRA spectrum after formic acid exposure; calculations for $1 / 3$ formic acid coverage; surface phase diagram; density of states for the different adsorption configurations (PDF)

\section{AUTHOR INFORMATION}

\section{Corresponding Authors}

Gregor B. Vonbun-Feldbauer - Institute of Advanced Ceramics, Hamburg University of Technology (TUHH), 21073 Hamburg, Germany; 10 orcid.org/0000-0002-93270450; Email: gregor.feldbauer@tuhh.de

Andreas Stierle - DESY NanoLab, Deutsches ElektronenSynchrotron (DESY), 22607 Hamburg, Germany; Department of Physics, University of Hamburg, 20355 
Hamburg, Germany; 이이.org/0000-0002-0303-6282;

Email: andreas.stierle@desy.de

\section{Authors}

Marcus Creutzburg - DESY NanoLab, Deutsches ElektronenSynchrotron (DESY), 22607 Hamburg, Germany; Department of Physics, University of Hamburg, 20355 Hamburg, Germany; (1) orcid.org/0000-0002-3639-6049

Kai Sellschopp - Institute of Advanced Ceramics, Hamburg University of Technology (TUHH), 21073 Hamburg, Germany; (i) orcid.org/0000-0002-0003-2075

Steffen Tober - DESY NanoLab, Deutsches ElektronenSynchrotron (DESY), 22607 Hamburg, Germany; Department of Physics, University of Hamburg, 20355 Hamburg, Germany

Elin Grånäs - DESY NanoLab, Deutsches ElektronenSynchrotron (DESY), 22607 Hamburg, Germany; (1) orcid.org/0000-0001-5247-7589

Vedran Vonk - DESY NanoLab, Deutsches ElektronenSynchrotron (DESY), 22607 Hamburg, Germany; (1) orcid.org/0000-0001-9854-1101

Wernfried Mayr-Schmölzer - Institute of Advanced Ceramics, Hamburg University of Technology (TUHH), 21073 Hamburg, Germany; 이이이.org/0000-0003-48349458

Stefan Müller - Institute of Advanced Ceramics, Hamburg University of Technology (TUHH), 21073 Hamburg, Germany

Heshmat Noei - DESY NanoLab, Deutsches ElektronenSynchrotron (DESY), 22607 Hamburg, Germany;

○ orcid.org/0000-0003-1294-3527

Complete contact information is available at: https://pubs.acs.org/10.1021/acs.jpclett.1c00209

\section{Author Contributions}

${ }^{\perp}$ M.C. and K.S. contributed equally to the paper.

\section{Notes}

The authors declare no competing financial interest.

\section{ACKNOWLEDGMENTS}

This project is funded by the Deutsche Forschungsgemeinschaft (DFG, German Research Foundation)-Projektnummer 192346071-SFB 986. We gratefully acknowledge excellent support at beamline ID03 of the European Synchrotron Radiation Facility (ESRF), especially Francesco Carlà and Helena Isern Herrera.

\section{REFERENCES}

(1) Baeza, A.; Guillena, G.; Ramón, D. J. Magnetite and MetalImpregnated Magnetite Catalysts in Organic Synthesis: A Very Old Concept with New Promising Perspectives. ChemCatChem 2016, 8, 49-67.

(2) de Smit, E.; Weckhuysen, B. M. The Renaissance of Iron-based Fischer-Tropsch Synthesis: On The Multifaceted Catalyst Deactivation Behaviour. Chem. Soc. Rev. 2008, 37, 2758-2781.

(3) Zhu, M.; Wachs, I. E. Iron-Based Catalysts for the HighTemperature Water-Gas Shift (HT-WGS) Reaction: A Review. ACS Catal. 2016, 6, 722-732.

(4) Albert, J.; Jess, A.; Kern, C.; Pöhlmann, F.; Glowienka, K.; Wasserscheid, P. Formic Acid-Based Fischer-Tropsch Synthesis for Green Fuel Production from Wet Waste Biomass and Renewable Excess Energy. ACS Sustainable Chem. Eng. 2016, 4, 5078-5086.

(5) Yasaka, Y.; Yoshida, K.; Wakai, C.; Matubayasi, N.; Nakahara, M. Kinetic and Equilibrium Study on Formic Acid Decomposition in
Relation to the Water-Gas-Shift Reaction. J. Phys. Chem. A 2006, 110, $11082-11090$.

(6) Dreyer, A.; Feld, A.; Kornowski, A.; Yilmaz, E. D.; Noei, H.; Meyer, A.; Krekeler, T.; Jiao, C.; Stierle, A.; Abetz, V.; et al. Organically Linked Iron Oxide Nanoparticle Supercrystals With Exceptional Isotropic Mechanical Properties. Nat. Mater. 2016, 15, 522-528.

(7) Jupille, J.; Thornton, G. Defects at Oxide Surfaces; Springer International Publishing, 2015.

(8) Rousseau, R.; Glezakou, V.-A.; Selloni, A. Theoretical Insights Into the Surface Physics and Chemistry of Redox-active Oxides. Nat. Rev. Mater. 2020, 5, 460-475.

(9) Feld, A.; Weimer, A.; Kornowski, A.; Winckelmans, N.; Merkl, J.P.; Kloust, H.; Zierold, R.; Schmidtke, C.; Schotten, T.; Riedner, M.; et al. Chemistry of Shape-Controlled Iron Oxide Nanocrystal Formation. ACS Nano 2019, 13, 152-162.

(10) Arndt, B.; Sellschopp, K.; Creutzburg, M.; Grånäs, E.; Krausert, K.; Vonk, V.; Müller, S.; Noei, H.; Feldbauer, G. B. V.; Stierle, A. Carboxylic Acid Induced Near-surface Restructuring of a Magnetite Surface. Comm. Chem. 2019, 2, 92.

(11) Gamba, O.; Noei, H.; Pavelec, J.; Bliem, R.; Schmid, M.; Diebold, U.; Stierle, A.; Parkinson, G. S. Adsorption of Formic Acid on the $\mathrm{Fe}_{3} \mathrm{O}_{4}(001)$ Surface. J. Phys. Chem. C 2015, 119, 2045920465.

(12) Xu, M.; Noei, H.; Buchholz, M.; Muhler, M.; Wöll, C.; Wang, Y. Dissociation of Formic Acid on Anatase $\mathrm{TiO}_{2}(101)$ Probed by Vibrational Spectroscopy. Catal. Today 2012, 182, 12-15.

(13) Buchholz, M.; Li, Q.; Noei, H.; Nefedov, A.; Wang, Y.; Muhler, M.; Fink, K.; Wöll, C. The Interaction of Formic Acid with Zinc Oxide: A Combined Experimental and Theoretical Study on Single Crystal and Powder Samples. Top. Catal. 2015, 58, 174-183.

(14) Cutting, R. S.; Muryn, C. A.; Thornton, G.; Vaughan, D. J. Molecular Scale Investigations of the Reactivity of Magnetite with Formic Acid, Pyridine, and Carbon Tetrachloride. Geochim. Cosmochim. Acta 2006, 70, 3593-3612.

(15) Parkinson, G. S. Iron Oxide Surfaces. Surf. Sci. Rep. 2016, 71, $272-365$

(16) Ritter, M.; Weiss, W. $\mathrm{Fe}_{3} \mathrm{O}_{4}(111)$ Surface Structure Determined by LEED Crystallography. Surf. Sci. 1999, 432, 81-94.

(17) Sala, A.; Marchetto, H.; Qin, Z.-H.; Shaikhutdinov, S.; Schmidt, T.; Freund, H.-J. Defects and Inhomogeneities in $\mathrm{Fe}_{3} \mathrm{O}_{4}(111)$ Thin Film Growth on Pt(111). Phys. Rev. B: Condens. Matter Mater. Phys. 2012, 86, 155430.

(18) Shimizu, T. K.; Jung, J.; Kato, H. S.; Kim, Y.; Kawai, M. Termination and Verwey Transition of the (111) Surface of Magnetite Studied by Scanning Tunneling Microscopy and Firstprinciples Calculations. Phys. Rev. B: Condens. Matter Mater. Phys. 2010, 81, 235429.

(19) Lennie, A. R.; Condon, N. G.; Leibsle, F. M.; Murray, P. W.; Thornton, G.; Vaughan, D. J. Structures of $\mathrm{Fe}_{3} \mathrm{O}_{4}(111)$ Surfaces Observed by Scanning Tunneling Microscopy. Phys. Rev. B: Condens. Matter Mater. Phys. 1996, 53, 10244-10253.

(20) Noh, J.; Osman, O. I.; Aziz, S. G.; Winget, P.; Brédas, J.-L. Magnetite $\mathrm{Fe}_{3} \mathrm{O}_{4}(111)$ Surfaces: Impact of Defects on Structure, Stability, and Electronic Properties. Chem. Mater. 2015, 27, 58565867.

(21) Li, X.; Paier, J.; Sauer, J.; Mirabella, F.; Zaki, E.; Ivars-Barceló, F.; Shaikhutdinov, S.; Freund, H.-J. Surface Termination of $\mathrm{Fe}_{3} \mathrm{O}_{4}(111)$ Films Studied by $\mathrm{CO}$ Adsorption Revisited. J. Phys. Chem. B 2018, 122, 527-533.

(22) Dementyev, P.; Dostert, K.-H.; Ivars-Barceló, F.; O’Brien, C. P.; Mirabella, F.; Schauermann, S.; Li, X.; Paier, J.; Sauer, J.; Freund, H.-J. Water Interaction with Iron Oxides. Angew. Chem., Int. Ed. 2015, 54, 13942-13946.

(23) Mirabella, F.; Zaki, E.; Ivars-Barceló, F.; Li, X.; Paier, J.; Sauer, J.; Shaikhutdinov, S.; Freund, H.-J. Cooperative Formation of LongRange Ordering in Water Ad-layers on $\mathrm{Fe}_{3} \mathrm{O}_{4}(111)$ Surfaces. Angew. Chem., Int. Ed. 2018, 57, 1409-1413. 
(24) Petitto, S. C.; Tanwar, K. S.; Ghose, S. K.; Eng, P. J.; Trainor, T. P. Surface Structure of Magnetite (111) under Hydrated Conditions by Crystal Truncation Rod Diffraction. Surf. Sci. 2010, 604, 1082-1093.

(25) Rim, K. T.; Eom, D.; Liu, L.; Stolyarova, E.; Raitano, J. M.; Chan, S.-W.; Flytzani-Stephanopoulos, M.; Flynn, G. W. Charging and Chemical Reactivity of Gold Nanoparticles and Adatoms on the (111) Surface of Single-Crystal Magnetite: A Scanning Tunneling Microscopy/Spectroscopy Study. J. Phys. Chem. C 2009, 113, 1019810205.

(26) Arndt, B.; Bliem, R.; Gamba, O.; van der Hoeven, J. E. S.; Noei, H.; Diebold, U.; Parkinson, G. S.; Stierle, A. Atomic Structure and Stability of Magnetite $\mathrm{Fe}_{3} \mathrm{O}_{4}(001)$ : An X-ray View. Surf. Sci. 2016, $653,76-81$.

(27) Nara, M.; Torii, H.; Tasumi, M. Correlation between the Vibrational Frequencies of the Carboxylate Group and the Types of Its Coordination to a Metal Ion: An ab Initio Molecular Orbital Study. J. Phys. Chem. 1996, 100, $19812-19817$.

(28) Wang, Y.; Wöll, C. IR Spectroscopic Investigations of Chemical and Photochemical Reactions on Metal Oxides: Bridging the Materials Gap. Chem. Soc. Rev. 2017, 46, 1875-1932.

(29) Würger, T.; Heckel, W.; Sellschopp, K.; Müller, S.; Stierle, A.; Wang, Y.; Noei, H.; Feldbauer, G. Adsorption of Acetone on Rutile $\mathrm{TiO}_{2}$ : A DFT and FTIRS Study. J. Phys. Chem. C 2018, 122, 1948119490.

(30) Kiss, J.; Frenzel, J.; Meyer, B.; Marx, D. Methanol Synthesis on $\mathrm{ZnO}(000 \overline{1})$. II. Structure, Energetics, and Vibrational Signature of Reaction Intermediates. J. Chem. Phys. 2013, 139, 044705.

(31) Giannozzi, P.; Baroni, S. Handbook of Materials Modeling; Springer: Dordrecht, 2005; pp 195-214.

(32) Chase, M. NIST-JANAF Thermochemical Tables, 4th ed.; American Institute of Physics, 1998.

(33) Chao, J.; Zwolinski, B. J. Ideal gas thermodynamic properties of methanoic and ethanoic acids. J. Phys. Chem. Ref. Data 1978, 7, 363377.

(34) Parkinson, G. S.; Mulakaluri, N.; Losovyj, Y.; Jacobson, P.; Pentcheva, R.; Diebold, U. Semiconductor-Half Metal Transition at the $\mathrm{Fe}_{3} \mathrm{O}_{4}(001)$ Surface Upon Hydrogen Adsorption. Phys. Rev. B: Condens. Matter Mater. Phys. 2010, 82, 125413. 